\title{
Photometric and spectroscopic study of candidate Be stars in the Magellanic Clouds
}

\author{
Paul KT ${ }^{1}$, Annapurni Subramaniam², Blesson Mathew ${ }^{2}$, \\ Ronald E. Mennickent ${ }^{3}$, and Beatriz Sabogal ${ }^{4}$ \\ ${ }^{1}$ Christ University,Bangalore, ${ }^{2}$ Indian institute of Astrophysics,Bangalore, ${ }^{3}$ University of \\ Concepcion, Chile, ${ }^{4}$ Universidad de los Andes, Colombia
}

\section{Introduction}

Mennickent et al. (2002) presented a catalogue of 1056 Be star candidates in the Small Magellanic cloud (SMC) by studying light curve variation using OGLE II data base. They classified these Be star candidates of the SMC in four categories: Type 1 stars showing outbursts (139 stars); Type 2 stars showing sudden luminosity jumps (154 stars); Type 3 stars showing periodic or near periodic variations (78 stars); Type 4 stars showing light curves similar to Galactic Be stars (658 stars). They suggested that Type 4 could be Be stars. On the other hand, they suggested that Type-3 stars may not be linked to the Be star phenomenon at all.

Based on a similar inspection of OGLE II data, Sabogal et al. (2005) classified Be candidates in the Large Magellanic Cloud (LMC) also as Type 1 (581 stars), Type 2 (150 stars), Type 3 (149 stars) and Type 4 stars (1468 stars). However most of the type 4 stars in LMC are found to be reddened and located parallel to the main sequence, this feature was not found in the same diagrams of the SMC. The photometric properties of Type 1 and Type 3 stars on the LMC are very different from those of the SMC. Thus, the various types of stars identified based on variability seem to differ between the LMC and the SMC.We have studied the near IR properties of various types of Be star candidates in the LMC and SMC by cross matching IRSF and OGLE II catalogs. We aim to study the properties of various types and compare them in the LMC and SMC, in particular, the type 4 stars. We also correlated the LMC and SMC Be star candidates with galactic counter parts through NIR colour-colour diagram. We also present results from a spectroscopic study of a 49 stars from Types 1, 2 and 3 in the SMC and 12 stars belonging to type 1 and type 3 in the LMC. The spectral features are used to identify their spectral class and to identify the nature of these stars, including the Be properties.

\section{Results}

In order to obtain their near-IR properties, we used the near-IR IRSF catalog (Kato et al. 2007; http://pasj.asj.or.jp/v59/n3/590315). The optically identified stars are crossmatched with near-IR IRSF calaloge to confirm its candidature in IRSF. The near-IR photometric magnitude in $\mathrm{J}, \mathrm{H}$ and $\mathrm{Ks}$ bands for the identified candidate stars were taken from IRSF database. These stars were used to study the near-IR properties of the various Types. Optical spectra were obtained for 61 candidate stars in the LMC and SMC. Observations were made during October 2002 using the $1.5 \mathrm{~m}$ CTIO Telescope. 56 Blue(3700$5500) \AA$ and 51 red (5700-7000) $\AA$ spectra were obtained with a resolution of $3.7 \AA$.

We cross correlated 1640 stars identified by Sabogal et al. (2005). Of these, 399 are Type 1 stars, 92 are Type 2 stars, 91 are Type 3 stars and 989 stars are Type 4 . The 

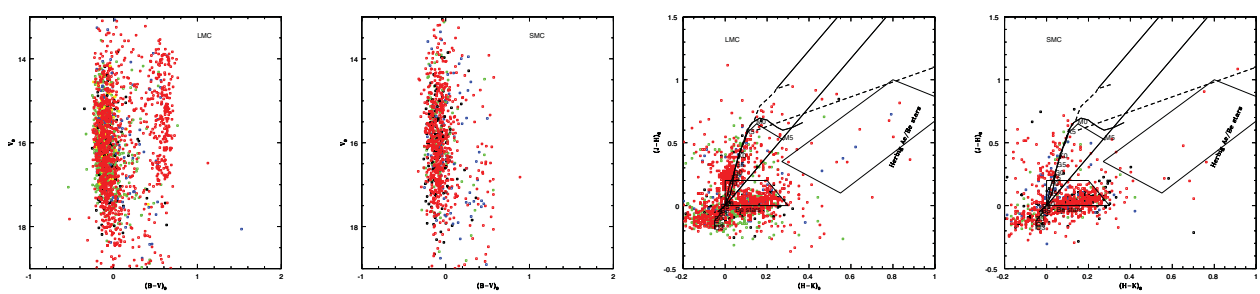

Figure 1. $\operatorname{CMDs}\left((\mathrm{B}-V)_{0}\right.$ vs $\left.\mathrm{V}_{0}\right)$ and $\operatorname{CCDs}\left((H-K)_{0} \operatorname{Vs}(J-H)_{0}\right)$ for the cross matched stars in the $\mathrm{LMC}$ and $\mathrm{SMC}$

SMC sample contains 841 stars, of which 89 are Type 1 stars, 131 are type 2 stars, 65 are Type 3 stars and 554 are Type 4 stars.

Adopting $R=A_{v} / E(B-V)=3.1$ on the basis of previously published values, colour magnitude diagrams with $(\mathrm{B}-V)_{0}$ vs $\mathrm{V}_{0}$ were plotted for the LMC and the SMC(type 1 to type 4 shown in different colours) We notice the presence of Type -4 stars parallel to the main sequence in the range of $\mathrm{B}-\mathrm{V}=0.4$ to $0.7 \mathrm{mag}$, in the $\mathrm{LMC}$, which is absent in the SMC. These figures suggest that the cross-identified stars have sampled all the types and this subset can be used to study the properties of various types in the near-IR. We estimated the dereddened $(\mathrm{J}-\mathrm{H})_{0}$ and $(\mathrm{H}-\mathrm{K})_{0}$ values of the cross matched sources. Reddening corrections were made using formulae given by (Bessell \& Brett 1988) by taking $\mathrm{E}(\mathrm{B}-\mathrm{V})=0.1 \mathrm{mag}$. Color-Color diagrams $(\mathrm{CCD})$, were plotted with $(H-K)_{0}$ Vs $(J-H)_{0}$ for all the types in the LMC and SMC. The CCD for the LMC shows that most of the stars are populated near the $(J-H)_{0} \sim 0.0$, with a small range in $(H-K)_{0}$, as a more or less horizontal band. We do also notice that some stars occupy a location above this band, on the MS, giving rise to a clumpy appearance. The stars in this clump are mostly Type 4 stars. The CCD for the SMC also shows the horizontal band like distribution, but the clumpy population is not found in the SMC. We identify a few sources with large near-IR excess in the LMC, belonging to Type 3 and Type 4 . SMC shows fewer sources with near-IR excess.

We compared the spectra of the Be candidate stars with those of the standard stars and the Be stars were found to be in the range from O5 to A3. We find that type 1, type 2 and type 3 stars have similar NIR properties in the LMC and in the SMC. Spectral analysis of selected stars from type 1, type 2 and type 3 shows that most of them belong to early spectral type (early A and B type). Spectra of most of the stars show $\mathrm{H}_{\alpha}$ emission. Hence we conclude that type 1, 2, 3 stars could be Be stars. Among the sample studied type 3 stars in the LMC have relatively less $\mathrm{H}_{\alpha}$ EW when compared to those in SMC. Type 2 stars are likely to be Be-stars and unlikely to be pre-MS stars, since they lack near IR excess. Type 4 stars in the LMC fall in two groups, one with nearIR properties similar to those of the Galactic Be stars, and a NEW GROUP with different nearIR properties. The type 4 stars in the SMC have nearIR properties similar to the Galactic Be stars.

\section{References}

Mennickent, R. E., Pietrzyński, G., Gieren, W., \& Szewczyk, O. 2002, A\&A 393, 887

Sabogal, B. E., Mennickent, R. E., Pietrzyński, G., \& Gieren, W. 2005, MNRAS 361, 1055 